Assessment Tool:Cancer (NAT:-C) is a validated tool to improve needs-based access to palliative care.

Aims To assess the feasibility and acceptability of conducting a definitive cRCT of the NAT:-C in primary care in terms of

1. Recruiting 4 GP practices and 40-60 patients (plus carers),

2. Uptake of NAT:-C,

3. Questionnaire completion rates,

4. Acceptability of study measures.

Methods A non-blinded cluster randomised trial with parallel process evaluation. Patients with active cancer were identified through cancer registries or in clinic and invited to participate and nominate a carer if they wanted to. Participants completed study questionnaires at baseline and 1, 3 and 6 months. Patients sought a $20 \mathrm{~min}$ appointment post-baseline. Patients, carers and GP practice staff were invited to participate in interviews/focus groups.

Results Five GP practices were approached and 4 recruited and trained in the use of the NAT:-C. Practices were cluster randomised $(1: 1)$. Forty-seven patients and 17 carers were recruited. Early recruitment problems were addressed by including a pre-paid expression of interest form with the study invitation. Recruitment rate was appropriate for a definitive multicentre trial. 44 (94\%) of patients received a study appointment, 42 (95\%) attended and $32(76 \%)$ received a NAT-guided consultation. Item completion rates of the proposed primary outcome measure the Supportive Care Needs Survey at baseline were 96.4\%. 15 patient interviews and 4 focus groups with GP practices were conducted. Participants supported the need for a definitive study and found all measures acceptable. Process evaluation informed effective refinement of study invitations.

Conclusion The feasibility trial indicated that the recruitment rate, intervention uptake and data collection were appropriate, with refinements, for a definitive cRCT is feasible.

\section{WHAT ARE THE MAIN CONCERNS OF PARTICIPANTS IN A DRUG TRIAL FOR CHRONIC BREATHLESSNESS?}

S Singh, N Lovell, D Yi, IJ Higginson. Cicely Saunders Institute, Department of Palliative Care, Policy and Rehabilitation, King's College London

\subsection{6/bmjspcare-2019-ASP.170}

Background Chronic breathlessness is a common, distressing advanced illness symptom. Previous work has synthesised patient concerns, but not specifically within a trial setting.

Aims To determine the main concerns of participants in a drug trial for chronic breathlessness and assess change during the trial.

Methods Data was collected as part of a multi-centre randomised placebo controlled, double-blind feasibility trial of Mirtazapine for patients with chronic breathlessness (ISRCTN 33236160). The question 'What have been your main concerns or problems over the past 3 days?' as part of the Integrated Palliative Outcomes Scale (IPOS) was asked at three time-points (baseline, day 14, day 28). Participants were able to provide up to three answers. A free text analysis was done, and concerns were categorised using the domains of total breathlessness. Results were compared across the time-points of the trial to assess for change.
Results 64 patients participated in the trial. $73 \%$ were male; median age 72 years (range 51-90). 63\% had a diagnosis of chronic obstructive pulmonary disease, 30\% interstitial lung disease, and $8 \%$ chronic heart failure or cancer. 267 responses were collected. The most commonly reported concerns were breathlessness ( $\mathrm{n}=46$ baseline, $\mathrm{n}=20$ day $14, \mathrm{n}=28$ day 28), fatigue ( $n=7$ baseline, $n=6$ day $14, n=6$ day 28 ) and mobility ( $n=4$ baseline, $n=7$ day $14, n=4$ day 28). Using the model of total breathlessness, $81 \%$ of concerns were categorised within the physical domain. $23 \%$ as psychological, $6 \%$ spiritual, and $3 \%$ social. 35/267 concerns showed overlap across multiple domain.

Conclusion Physical concerns, specifically breathlessness, fatigue and mobility were most common. Concerns appeared to remain stable during the trial period. It is crucial that outcome measures which reflect the concerns of patients are selected.

\section{ESTABLISHING RESEARCH PRIORITIES TO IMPROVE THE MANAGEMENT OF PATIENTS WITH ADVANCED HEART FAILURE FROM THE PEOPLE THAT MATTER}

Amy Gadoud. Lancaster University, University of Bristol, University of Oxford, University of Cambridge

\subsection{6/bmjspcare-2019-ASP.171}

Background Patients with advanced heart failure have a significant symptom burden and a range of palliative care needs but these are often unmet. Research priorities have traditionally been set by funders and researchers and focus on active treatment and palliative care research is not prioritised. Involving patients in the process can lead to more valid, credible and relevant research findings. This project sets out to establish shared research priorities for advanced heart failure from the patient, carer and clinician perspective, as a James Lind Alliance partnership.

Methods A steering group of people directly affected by advanced heart failure including patients, carers and clinicians is overseeing the project. The results of an initial online survey of priorities are presented here. A review of the literature will be carried out to identify where priorities have already been addressed, and where gaps exist. Submitted priorities will be sorted by a second online survey to generate a shorter list for discussion at a final workshop in early 2019, where a 'Top Ten' will be agreed. The 'Top Ten' will be disseminated widely to researchers and funders to influence the advanced heart failure research agenda.

Results Preliminary results of the online survey of 180 participants has demonstrated palliative research questions are a priority for this group. Themes include planning for the future, coordination of care, communication and supporting family members and carers. Comorbidity was recognised as common. Symptom control was also prioritised, including symptoms under-recognised by clinicians such as fatigue, and psychological issues like depression, loneliness and poor cognition.

Conclusion Preliminary results indicate new insight into what matters to people and what they would like to see researched. The palliative care research community should be ready to take up the challenge of exploring these unanswered questions.

Funding NIHR School of Primary Care Research. 